\title{
Dynamic Transesophageal Echocardiographic Parameters for Predicting Fluid Responsiveness in Mechanically Ventilated Patients
}

\section{Yaru Li}

Peking University People's Hospital

Luyang Jiang ( $\sim$ jiangly1018@hotmail.com )

Peking University People's Hospital https://orcid.org/0000-0002-4341-4444

\section{Yi Feng}

Peking University People's Hospital

Hai Ting

Peking University People's Hospital

\section{Guang Wang}

Peking University People's Hospital

\section{Lu Wang}

Peking University People's Hospital

Dou Dou

Peking University People's Hospital

\section{Research}

Keywords: fluid responsiveness FR, transesophageal echocardiography TEE, superior vena cava SVC, stroke volume variation SVV

Posted Date: September 14th, 2021

DOI: https://doi.org/10.21203/rs.3.rs-882544/v1

License: (a) (i) This work is licensed under a Creative Commons Attribution 4.0 International License. Read Full License 


\section{Abstract}

Background: The aim was to investigate the feasibility of the dynamic transesophageal echocardiographic parameters to predict fluid responsiveness in mechanically ventilated patients.

Methods: In the prospective study, a total of 60 patients scheduled for elective general surgery under mechanical ventilation were enrolled. All patients received $10 \mathrm{ml} / \mathrm{kg}$ Ringer's lactate. The data including central venous pressure (CVP), cardiac index (CI), stroke volume variation (SVV), SVC-Cl, E velocity, and the ratio of E/e' was recorded before and after fluid challenge. Patients were classified as Responders (FR group) if their $\mathrm{Cl}$ increased by at least $15 \%$ after fluid challenge.

Results: 25/52(48\%) were Responders and 27 were non-Responders (52\%). The SVC-Cl was higher in the Responders ( $41.90 \pm 11.48 \%$ vs $28.92 \pm 9.05 \%, P \otimes 0.01)$. SVC-Cl was significantly correlated with $\triangle \mathrm{Cl}$ $(\mathrm{r}=0.568, P \otimes 0.01)$. The area under the ROC curve (AUROC) of SVC-Cl was 0.838 (95\% Cl: $0.728 \otimes 0.947, P]$ 0.01 ) with the optimal cut-off value of $39.4 \%$ (sensitivity $64 \%$, specificity $92.6 \%$ ). The best cut-off value for SVV was $12.5 \%$ (sensitivity $40 \%$, specificity $89 \%$ ) with the AUROC of $0.68(95 \% \mathrm{Cl} 0.53 \otimes 0.826, P \otimes 0.05)$.

Conclusion: The SVC-Cl and SVV can predict fluid responsiveness effectively in mechanically ventilated patients. And SVC-Cl is superior in predicting fluid responsiveness compared with SVV.

Trial registration: Chinese Clinical Trial Registry, ChiCTR2000034940, Registered 25 July 2020, https://www.chictr.org.cn/index.aspx

\section{Introduction}

Appropriate infusion strategy is the main treatment in perioperative patients. According to the FrankStaring curve, an increase in preload can increase the patient's stroke volume until it reaches the flat phase of the curve [1-4]. Fluid overloading may be deleterious by leading to systemic and pulmonary edema. To avoid fluid overload, it is critical to assess fluid responsiveness. Fluid responsiveness (FR) was defined by a $15 \%$ increase of the $\mathrm{CO}, \mathrm{Cl}$, or SV after fluid administration [5]. Only $50 \%$ of patients are fluid responders in ICU and operation room. There are some dynamic parameters such as stroke volume variation and pulse pressure variation that could predict FR $[6,7]$.

Recently, the development of Point-of-Care ultrasound (POCUS) makes it possible to predict FR visually. Moreover, Perioperative transesophageal echocardiography (TEE) or transthoracic echocardiography (TTE) monitoring can provide real-time hemodynamic information. Several studies found that respiratory diameter variation of great veins direct connected to right atrial chamber may be excellent to predict fluid responsiveness [8-10]. Under mechanical ventilation, the superior vena cava (SVC) regularly expands or collapses. During inhalation, the intrathoracic pressure increases, hence SVC is directly compressed and collapses as an intrathoracic vein. On the contrary, SVC expands during expiration [11]. The periodic changes of the SVC diameter are more obvious in hypovolemic patients. 
E velocity refers to the peak early filling velocity of rapid trans-mitral flow as the mitral valve opens during early diastole and it reflects the left ventricular diastolic function. The e' is the mitral annular tissue early diastole velocity. The ratio of $\mathrm{E} / \mathrm{e}^{\prime}$ is considered to be one of the most reproducible echocardiographic parameters to estimate pulmonary capillary wedge pressure (PCWP), which is regarded as a quantitative assessment of LV preloading conditions [14].

The primary aim of this study was to investigate the feasibility of the dynamic TEE parameters including SVC-Cl, E or e' velocities, the ratio of E/e' to predict FR in mechanically ventilated patients, and the secondary objective was to compare the predictive value of those TEE parameters and conventional indices including SVV and CVP.

\section{Materials And Methods}

\subsection{Study Design}

The prospective diagnostic study was conducted in the department of anesthesiology of People's Hospital of Peking University and was approved by the Institutional Review Board of our institution (Ethics Committee of Peking university people's hospital 2020PHB139-01). The study was registered at the Chinese clinical trial registry (ChiCTR2000034940). Written informed consents were obtained from all participants. Patients who underwent general anesthesia with tracheal intubation for abdominal surgery were consecutively included. Inclusion criteria were: age at 18-70 years old, ASAI-III, NYHA I-II grade. Exclusion criteria included TEE contradictions such as gastroduodenal ulcer, the history of esophagus operation, esophagus fundus ventricular varication, and arrhythmia, left ventricle $E F<55 \%$, average $E / e^{\prime}$ $>14$ or $\mathrm{e}^{\prime}$ average $<9 \mathrm{~cm} / \mathrm{s}$ at baseline, severe valvular disease.

Upon arrival in the operating room, all patients were monitored with pulse oximetry and electrocardiograph, radial artery catheterization connecting the FloTrac ${ }^{\mathrm{TM}} /$ Vigileo $^{\mathrm{TM}}$ ( Edwards Lifesciences,USA). Anesthesia induction was done with midazolam $(1 \mathrm{mg})$, sufentanil $(0.25 \mu \mathrm{g} / \mathrm{kg})$, etomidate $(0.3 \mathrm{mg} / \mathrm{kg})$, rocuronium $(0.6-1 \mathrm{mg} / \mathrm{kg})$ and maintained with sevoflurane inhalation. All patients were continuous monitored with BIS (range of 40-60). Patients were mechanically ventilated in volumecontrolled ventilation: $\mathrm{V}_{\mathrm{T}}$ of $8 \mathrm{ml} / \mathrm{kg}$, respiratory rate of $12 \mathrm{breath} / \mathrm{min}$, no PEEP was applied.

After induction, the TEE probe (6TC-RS GE Medical Horton,Norway)was inserted orally. During the whole measurement, the patients' position remained unchanged.

\subsection{Data collection}

Group 1 basic hemodynamic data: MAP, $H R$, and $C V P$;

Group 2 functional hemodynamic data using FloTrac: $\mathrm{Cl}$ and $\mathrm{SVV}$;

Group 3 echocardiographic data using TEE: SVC-Cl, E velocity, E/e', LVEDd (left ventricular end-diastole diameter). 
SVC-Cl (via M-mode), E velocity (via pulse wave doppler), $e^{\prime}\left(e^{\prime}=e^{\prime}{ }_{\text {lateral }}+e^{\prime}\right.$ septal $/ 2$, via tissue doppler from both lateral and septal side of mitral annular), LVEDd (via M-mode).

\subsection{Fluid responsiveness}

Fluid challenge: A fluid challenge was conducted with $10 \mathrm{ml} / \mathrm{kg}$ of a Ringer's lactate for $15-20$ minutes.

$\triangle \mathrm{Cl}$ was calculated as follows: $\triangle \mathrm{Cl}=\left(\mathrm{Cl}_{\text {after }}-\mathrm{Cl}_{\text {baseline }}\right) / \mathrm{Cl}_{\text {baseline }}{ }^{*} 100 \%$. Patients were classified as Responders (FR group: $\triangle \mathrm{Cl} \geq 15 \%$ ) and Non-Responders (NR group: $\triangle \mathrm{Cl} \otimes 15 \%$ ).

We also calculated the $\triangle$ LVEDd using the formula analogous to $\triangle \mathrm{Cl}$ :

$\triangle \operatorname{LVEDd}=\left(\operatorname{LVEDd}_{\mathrm{after}^{-}}\right.$LVEDd $\left._{\text {baseline }}\right) / \operatorname{LVIDd}_{\text {baseline }}{ }^{\star 100 \%}$

\subsection{Data measurements}

\subsubsection{SVC-Cl measurement}

After tracheal intubation, we passed the TEE probe into the mid-esophagus (ME) position. The transducer angle was rotated forward from $90^{\circ}$ to $110^{\circ}$, to obtain the ME Bicaval View. In this view, the superior vena cava (SVC) and the right atrium (RA) can be well imaged. The SVC diameter was measured approximately $2 \mathrm{~cm}$ from the junction with RA using the M-mode. Take the M-mode cursor to the junction and perpendicular to the SVC and obtain the inner diameter within a single respiratory cycle (Fig. 1) [15]. Measure the maximum and minimum diameter over a single respiratory cycle. SVC-Cl was calculated as follows: $S V C-C l=\left(S V C_{\max }-S V C_{\min }\right) / S V C_{\max }{ }^{*} 100 \%$. Echocardiographic variables were derived from the US machine (Vivid 7 Pro, GE Vingmed Ultrasound AS, Horten, Norway). All measurements were made three times and the average was used for statistical analysis.

\subsubsection{LVEDd measurement}

In the trans-gastric left ventricular (LV) short-axis view at the midpapillary level, LV inner diameter could be measured using M-mode imaging [16].

\subsubsection{E velocity and $\mathrm{E} / \mathrm{e}^{\prime}$ measurement}

At the ME four-chamber view, position the pulse wave (PW) Doppler sample volume between mitral leaflet tips, and adjust the sample volume to align with the blood flow, then obtain the optimal image of the $E$ wave. At the same view, position the tissue Doppler (TDI) sample volume both at lateral and septal basal regions of mitral annular to acquire $e_{\text {lateral }}^{\prime}$ and $e_{\text {septal. }}^{\prime}$. The average $e^{\prime}$ velocity can be computed: $e_{\text {average }}^{\prime}=$ $\left(e_{\text {septal }}^{\prime}+e_{\text {lateral }}^{\prime}\right) / 2[17]$.

All measurements were performed by a National Board of qualified Echocardiography anesthesiologist strictly following the relevant guidelines [15-18]. 


\subsection{Statistical analysis}

For continuous variables, data were expressed as mean \pm SD (normality distribution) or median with interquartile range (non-normality distribution). For categorical variables, percentages were calculated and the normality distribution was assessed by the Shapiro-Wilk normality tests, and comparisons of percentages were performed with Fisher's exact test. The differences between Responders and NonResponders were assessed using the Mann-Whitney U-test or Student's t-test. Spearman's rank method was performed to test linear correlations between $\triangle \mathrm{LVEDd}$ and $\triangle \mathrm{Cl}$, basic SVC-Cl, and $\triangle \mathrm{Cl}$.

To determine the ability to predict fluid responsiveness, Receiver operating characteristics (ROC) curves were generated and the area under the ROC curve (AUROC) was calculated. All P -values were two-tailed and a P-value $<0.05$ was considered significant. All statistical analyses were performed with IBM SPSS Statistics 26.0 (IBM, Somers, NY, USA)

\subsection{Sample size}

Medcalc software (Windows 19.4, Ostend, Belgium) was used to calculate the sample size. According to the pilot study, we assume the AUROC of SVC-Cl was 0.75 , with an a error of 0.05 and power of 0.9 , and the ratio of sample size in the FR/NR group of 1.26 patients were required for each group. Considering dropout, we planned to recruit 60 patients finally.

\section{Results}

\subsection{Patients' characteristics}

60 patients were enrolled over an 8-month period (from August 2020 to May 2021). 8 patients were excluded due to the following reasons: consent refused (1case), using vasopressors due to hypotension ( 2 cases), arrhythmia ( 3 cases), and poor SVC image ( 1 case), Figure 2 showed the flowchart of enrollment. Consequently, 52 patients completed the study including colorectal surgery $(n=22)$, hepatectomy $(n=12)$ and pancreaticoduodenectomy $(n=18)$. There were 25 Fluid Responders and 27 NonResponders. The general characteristics of all the patients and comparisons between FR and NR at baseline are shown in Table 1. No differences were found between groups. All data between groups before and after fluid challenge are presented in Table 2.

Table 1 Baseline characteristics between the FR and NR group 


\begin{tabular}{|c|c|c|c|}
\hline Group & $\begin{array}{l}\text { Overall } \\
(n=52)\end{array}$ & $\begin{array}{l}\mathrm{FR} \\
(\mathrm{n} \otimes 25)\end{array}$ & $\begin{array}{l}\mathrm{NR} \\
(\mathrm{n} \otimes 27)\end{array}$ \\
\hline Gender (M/F区 & $24 / 28$ & $12 / 13$ & $12 / 15$ \\
\hline Age $[M(\mathrm{Q}) \mathrm{y}]$ & $57(49,63)$ & $58(39,63)$ & $56(51,65 \rrbracket$ \\
\hline $\mathrm{BMI}\left(x \pm s \mathrm{~kg} / \mathrm{m}^{2}\right)$ & $23.7 \pm 3.6$ & $24.6 \pm 4.2$ & $22.9 \pm 3.0$ \\
\hline ASA (I/II/IIII & $20 / 28 / 4$ & $10 / 13 / 2$ & $10 / 15 / 2$ \\
\hline NYHA (I/II) & $23 / 29$ & $11 / 14$ & $12 / 15$ \\
\hline
\end{tabular}

Abbreviations: BMI, body mass index; ASA, American Society of Anesthesiologists; NYHA, New York Heart Association

Table 2 Hemodynamic and echocardiographic data before and after fluid challenge

\begin{tabular}{|lllll|}
\hline & FR $(\mathrm{n}=25)$ & \multicolumn{3}{l|}{ NR $(\mathrm{n}=27)$} \\
\cline { 2 - 5 } & Baseline & After & Baseline & After \\
\hline MAP (mmHg) & $80.3 \pm 12.0$ & $82.2 \pm 12.7$ & $86.2 \pm 14.6$ & $79.0 \pm 13.3$ \\
\hline HR (Bpm) & $68.4 \pm 12.9$ & $65.7 \pm 12.2$ & $72.7 \pm 15.3$ & $59.2 \pm 9.0$ \\
\hline SVC-Cl (\%) & $41.9 \pm 11.5^{\mathrm{a}}$ & $29.5 \pm 8.4^{\mathrm{b}}$ & $28.9 \pm 9.0$ & $24.9 \pm 9.7$ \\
\hline SVV (\%) & $11.6 \pm 3.2^{\mathrm{a}}$ & $6.7 \pm 3.2^{\mathrm{b}}$ & $9.5 \pm 2.9$ & $7.0 \pm 2.5$ \\
\hline E velocity (m/s) & $0.50 \pm 0.18^{\mathrm{a}}$ & $0.70 \pm 0.14$ & $0.66 \pm 0.17$ & $0.69 \pm 0.21$ \\
E/e' & $7.27 \pm 2.32$ & $9.01 \pm 2.97$ & $8.76 \pm 3.28$ & $8.90 \pm 4.65$ \\
\hline CVP (mmHg) & $5.7 \pm 3.2$ & $7.8 \pm 4.3^{\mathrm{b}}$ & $5.8 \pm 3.1$ & $8.1 \pm 4.5^{\mathrm{c}}$ \\
\hline
\end{tabular}

Abbreviations: MAP, mean arterial pressure; HR, heart rate; SVC-Cl, superior vena cava collapsibility index; SVV, stroke volume variation; CVP, central venous pressure

${ }^{a} P \otimes 0.05$ compared with Non-Responders, ${ }^{b} P \otimes 0.05$ compared with baseline in FR group, ${ }^{c} P \otimes 0.05$ compared with baseline in NR group

3.2 Echocardiographic data

$\triangle \mathrm{Cl}$ was significantly correlated with $\triangle$ LVEDd in all subjects $(r=0.643, P \otimes 0.05$, Table 3 , Figure $3 A)$.

Table 3 The analysis of $\triangle \mathrm{Cl}$ and $\triangle$ LVEDd between two groups 


\begin{tabular}{|c|c|c|c|c|c|}
\hline & $\begin{array}{l}F R \\
(n=25)\end{array}$ & $\begin{array}{l}N R \\
(n=52)\end{array}$ & $\begin{array}{l}\text { Overall } \\
(n=52)\end{array}$ & $r$ & $\begin{array}{l}\text { Abbreviations: } \mathrm{Cl} \text {, cardiac index; LVEDd, } \\
\text { left ventricular end-diastole diameter }\end{array}$ \\
\hline$\triangle \mathrm{Cl}(\%)$ & $22.1 \pm 7.7$ & $5.1 \pm 5.0$ & $13.3 \pm 10.7$ & \multirow[t]{2}{*}{0.643} & \multirow{2}{*}{ 3.2.1 SVC-Cl analysis } \\
\hline$\triangle$ LVEDd $(\%)$ & $12.1 \pm 4.3$ & $5.9 \pm 2.3$ & $8.9 \pm 4.7$ & & \\
\hline
\end{tabular}

$0.568, P \otimes 0.01$; Figure 3B). The basic SVC-Cl was higher in the Responders compared with Non-

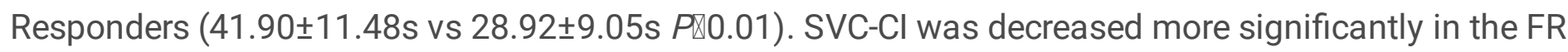
group compared with the NR group after fluid challenge.

\subsubsection{E velocity and $\mathrm{E} / \mathrm{e}^{\prime}$ analysis}

The E velocity was slightly correlated with $\triangle \mathrm{Cl}(\mathrm{r}=-0.372, P \otimes 0.01)$ and $\mathrm{E}$ velocity was lower in the FR group $(P \otimes 0.05)$. No correlation was found between $\mathrm{E} / \mathrm{e}^{\prime}$ and $\triangle \mathrm{Cl}(P \otimes 0.05)$, and there was no significant difference of $\mathrm{E} / \mathrm{e}^{\prime}$ between two groups. (Pख0.05).

\subsection{Functional hemodynamic data}

SVV was correlated slightly with $\triangle \mathrm{Cl}(\mathrm{r}=0.291, P \otimes 0.05 \mathrm{~A}$; Figure $3 \mathrm{C})$ and the $\mathrm{SVV}$ in the Responders was higher than Non-Responders too. (11.3 \pm 3.18 vs $9.52 \pm 2.94 P \otimes 0.05)$.

\subsection{Basic hemodynamic data}

CVP was not correlated with $\triangle \mathrm{Cl}$. There was no difference in basic CVP, HR, and MAP between the two groups. Overall, after fluid challenge, the HR decreased and CVP increased ( $P \otimes 0.05)$.

\subsection{ROC curve analysis}

The best cut-off value of SVC-Cl was $39.4 \%$ with $64 \%$ sensitivity and $92.6 \%$ specificity. The AUROC of SVC-Cl was $0.838(95 \% \mathrm{Cl}: 0.728 \otimes 0.947, P \otimes 0.01)$. SVV had a sensitivity of $40 \%$, a specificity of $89 \%$ to predict FR at a cut-off value of $12.5 \%$ and the AUROC was $0.68(95 \% \mathrm{Cl} 0.53 \otimes 0.826, P \otimes 0.05)$. The AUROC of CVP was 0.462(Pख0.05). The results of ROC analysis are shown in Figure 4.

\section{Discussion}

Our prospective study found that both SVC-Cl and SVV were reliable to predict fluid responsiveness in mechanically ventilated patients and SVC-Cl showed better accuracy than SVV regarding the area under curve of ROC. However, the value of CVP, E velocity, and E/e' to assess FR was doubtful.

First of all, $\triangle \mathrm{Cl}$ was significantly correlated with $\triangle$ LVEDd, which means the increase of cardiac output (measured by FloTrac) was consistent with the increase of LVEDd (measured using TEE), which was also the theoretical basis of our study. $\triangle \mathrm{Cl}$ and $\triangle \mathrm{LVEDd}$ are positively correlated in the FR group, which means the underline mechanism of FR is the response of left ventricular end-diastolic volume to rapid infusion. 
The SVC diameter is determined by blood volume and the external gradient pressure which is pleural pressure induced by positive-pressure ventilation. while volume is insufficient, suddenly increased intrathoracic pressure during inhalation is greater than the inner-vascular pressure and the vessel collapses consequently. Accordingly, the collapse of the SVC may reflect the blood volume in ventilated patients. Vieillard-Baron A's classic study [10] defined fluid responsiveness by an increase in $\mathrm{Cl}$ greater than or equal to $11 \%$ and the optimal cut-off value of SVC-Cl for predicting FR was $36 \%$. Recommend by recent studies, we assumed $\triangle \mathrm{Cl} \geq 15 \%$ as the standard of the $F R$, the ratio of $F R$ to NR was approximately $1: 1$, which was consistent with previous studies.

We found that the basic SVC-Cl of the FR group was greater than the NR group significantly. Moreover, the reduction in SVC-Cl after fluid challenge in the FR group was greater than that in the NR group, which indirectly reveals that extra fluid administration may not increase the effective circulating blood volume in Non-Responders. The inner diameter of SVC not only depends on the volume but also is related to pulmonary compliance. All patients in our study did not have any pulmonary diseases such as COPD or ARDS, so lung compliance had little effect on the results.

SVV is considered to be a reliable predictor of fluid responsiveness, but it has some acknowledged limitations: it is not suitable for pneumoperitoneum, arrhythmia, spontaneous breathing, and vasopressor use $[19,20]$. Based on the algorithm, the accuracy of SVV also depends on the waveform of the peripheral radial artery. At the same time, the application of SVV requires a high tidal volume of more than $8 \mathrm{ml} / \mathrm{kg}$ which may be contradictory to the lung-protective ventilation strategy. Compared with SVV, SVC is not affected by arrhythmia, intra-abdominal pressure, or vasoactive drugs. The cut-off value for SVV to predict FR was $12.5 \%$ in our research, which is close to the $13 \%$ threshold recommended by FloTrac instruction.

Preload is defined clinically as the left ventricular volume at end-diastole and it is estimated using several indirect methods such as left ventricular filling pressure (LVFP), pulmonary capillary wedge pressure (PCWP), and other indices related to LV diastolic dysfunction [21]. The importance of PCWP is that pressure presents the Left atrial (LA) pressure during end diastole and provides a means of measuring left ventricular preload. But PCWP is only can be directly measured via Swan-Ganz Catheter. E velocity refers to the peak early filling velocity of rapid trans-mitral flow as the mitral valve opens during diastole and it reflects the left ventricular diastolic function [18,22]. Our results showed the $E$ velocity was lower significantly in the FR group. E wave was determined by driving force between LA and LV, the compliance both of LA and LV. Due to the LA / LV compliance is decreasing with age, it's difficult to assume that the $\mathrm{E}$ velocity could predict $\mathrm{FR}$ directly. The ratio of $\mathrm{E} / \mathrm{e}$ ' is considered to be one of the most reproducible echocardiographic parameters to estimate PCWP [23]. But our study showed that E/e' couldn't discriminate fluid responsiveness effectively. It is probably because the E velocity is affected by several factors other than left ventricular diastole function and cardiac output [18]. Consequently, it needs advanced study to assess FR using E velocity or E/e' exclusively. 
Precise real-time measurement of continuous LV preload remains a challenging problem. POCUS becomes a routine bedside monitoring in the operating room and intensive care unit. Considering the study design, we placed TEE probe into the patients' body for a while, which could provide information before and after fluid administration. However, our long-time goal is to integrate the SVC-Cl measurement into bedside POCUS monitoring to evaluate the preload of critical patients quickly. It will provide a noninvasive and rapid FR assessment method. We assume that the greater the SVC-Cl, the greater the increase of cardiac output after rapid infusion. If the patient's basic SVC-Cl is less than $39.4 \%$, it means that rapid infusion will not increase cardiac output with potential harmful effects.

There are several limitations in our study. First, to avoid the potential deleterious effect of the rapid fluid administration, we excluded the patients who order than 70 years old, which were more necessary to assess FR and adopt target-directed fluid therapy. Second, due to the study design, we didn't analyze the influence of pneumoperitoneum or body position on assessing fluid responsiveness using those indices. Third, TEE is a minimally invasive procedure and it's reported that TEE-related complications range from $0.2-0.5 \%$ [24]. Otherwise, it requires professional training and experience to perform the TEE procedure.

\section{Conclusions}

The superior vena cava collapsibility index (SVC-CI) and SVV can predict fluid responsiveness effectively in mechanically ventilated patients. And SVC-Cl is superior in predicting fluid responsiveness compared with SVV in our study.

The E velocity and E/e' ratio couldn't predict fluid responsiveness and it needs advanced study to assess fluid responsiveness using E velocity or E/e' exclusively.

\section{Abbreviations}

CVP central venous pressure; $\mathrm{Cl}$ cardiac index; SVV stroke volume variation; FR fluid responsiveness; AUROC area under the Receiver Operating Characteristic curve; TEE transesophageal echocardiography; PCWP pulmonary capillary wedge pressure; LVEDd left ventricular end diastole diameter; POCUS point of care ultrasound;

\section{Declarations}

\section{Author's contributions}

Luyang Jiang and Yi Feng designed the study;

Luyang Jiang performed the ultrasound measurements and revised the manuscript;

Yaru Li enrolled the participants, participated in statistical analysis, and wrote the original draft; 
Lu Wang and Hai Ting participated in data collection, manuscript revision;

Dou Dou and Wang Guang participated in data collection, data analysis. All authors verified the data they contributed;

All authors approved the final manuscript for submission.

\section{Acknowledgement}

The author thanks Dr. Ran Zhang for providing assistance.

\section{Funding}

None.

\section{Availability of data and materials}

The datasets supporting the conclusions of this article are included within the article.

\section{Ethics approval and consent to participate}

This study was approved by the Ethics Committee of Peking university people's hospital (2020PHB13901) and registered at the Chinese clinical trial registry (ChiCTR2000034940).

\section{Competing Interests}

All authors disclosed that they do not have any conflicts of interest regarding this study.

ORCID ID

Luyang Jiang ID https://orcid.org/0000-0002-4341-4444

\section{References}

1. Cecconi M, Hofer C, Teboul JL, Pettila V, Wilkman E, Molnar Z, Della Rocca G, Aldecoa C, Artigas A, Jog S, Sander M, Spies C, Lefrant JY, De Backer D, Investigators F, Group ET. Erratum to: Fluid challenges in intensive care: the FENICE study: A global inception cohort study. Intensive Care Med. 2015;41(9):1737-38. https://doi.org/10.1007/s00134-015-3850-x.

2. Marik PE. Techniques for assessment of intravascular volume in critically ill patients. J Intensive Care Med. 2009;24(5):329-37. https://doi.org/10.1177/0885066609340640.

3. Frank O. Zur Dynamik des Herzmuskels. Z Biol 1895; 32: 370.

4. Starling EH: Linacre Lecture on the Law of the Heart. 1918.

5. Cecconi M, De Backer D, Antonelli M, Beale R, Bakker J, Hofer C, Jaeschke R, Mebazaa A, Pinsky MR, Teboul JL, Vincent JL, Rhodes A. Consensus on circulatory shock and hemodynamic monitoring. 
Task force of the European Society of Intensive Care Medicine. Intensive Care Med. 2014;40(12):1795-815. https://doi.org/10.1007/s00134-014-3525-z.

6. Michard F, Teboul JL. Predicting fluid responsiveness in ICU patients: a critical analysis of the evidence. Chest. 2002;121(6):2000-8. https://doi.org/10.1378/chest.121.6.2000.

7. Cherpanath TG, Hirsch A, Geerts BF, Lagrand WK, Leeflang MM, Schultz MJ, Groeneveld AB. Predicting Fluid Responsiveness by Passive Leg Raising: A Systematic Review and Meta-Analysis of 23 Clinical Trials. Crit Care Med. 2016;44(5):981-91. https://doi.org/10.1097/CCM.0000000000001556.

8. Wu CY, Cheng YJ, Liu YJ, Wu TT, Chien CT, Chan KC. Predicting stroke volume and arterial pressure fluid responsiveness in liver cirrhosis patients using dynamic preload variables: A prospective study of diagnostic accuracy. Eur J Anaesthesiol. 2016;33(9):645-52. https://doi.org/10.1097/EJA.0000000000000479.

9. Zlicar M, Novak-Jankovic V, Blagus R, Cecconi M. Predictive values of pulse pressure variation and stroke volume variation for fluid responsiveness in patients with pneumoperitoneum. J Clin Monit Comput. 2018;32(5):825-32. https://doi.org/10.1007/s10877-017-0081-4.

10. Vieillard-Baron A, Chergui K, Rabiller A, Peyrouset O, Page B, Beauchet A, Jardin F. Superior vena caval collapsibility as a gauge of volume status in ventilated septic patients. Intensive Care Med. 2004;30(9):1734-9. https://doi.org/10.1007/s00134-004-2361-y.

11. Bubenek-Turconi I, Hendy A, Bi S, Dr?gan A, Chioncel O, VI L, Moro?anu B, lliescu VA. The value of a superior vena cava collapsibility index measured with a miniaturized transoesophageal monoplane continuous echocardiography probe to predict fluid responsiveness compared to stroke volume variations in open major vascular surgery: a prospective cohort study. J Clin Monit Comput. 2020;34(3):491-9. https://doi.org/10.1007/s10877-019-00346-4.

12. Cheng Z, Yang QQ, Zhu P, Feng JY, Zhang XB, Zhao ZB. Transesophageal Echocardiographic Measurements of the Superior Vena Cava for Predicting Fluid Responsiveness in Patients Undergoing Invasive Positive Pressure Ventilation. J Ultrasound Med. 2019;38(6):1519-25. https://doi.org/10.1002/jum.14839.

13. Vignon P. Evaluation of fluid responsiveness in ventilated septic patients: back to venous return. Intensive Care Med. 2004;30(9):1699-701.

14. Mersch M, Schmidt C, Zarbock A. Echo physiology: the transesophageal echo probe as a noninvasive Swan-Ganz catheter. Curr Opin Anaesthesiol. 2016;29(1):36-45. https://doi.org/10.1097/ACO.0000000000000277.

15. Hahn RT, Abraham T, Adams MS, Bruce CJ, Glas KE, Lang RM, Reeves ST, Shanewise JS, Siu SC, Stewart W, Picard MH. Guidelines for performing a comprehensive transesophageal echocardiographic examination: recommendations from the American Society of Echocardiography and the Society of Cardiovascular Anesthesiologists. J Am Soc Echocardiogr. 2013;26(9):921-64. https://doi.org/10.1016/j.echo.2013.07.009. 
16. Lang RM, Badano LP, Mor-Avi V, Afilalo J, Armstrong A, Ernande L, Flachskampf FA, Foster E, Goldstein SA, Kuznetsova T, Lancellotti P, Muraru D, Picard MH, Rietzschel ER, Rudski L, Spencer KT, Tsang W, Voigt JU. Recommendations for cardiac chamber quantification by echocardiography in adults: an update from the American Society of Echocardiography and the European Association of Cardiovascular Imaging. J Am Soc Echocardiogr. 2015;28(1):1-39.e14. https://doi.org/10.1016/j.echo.2014.10.003.

17. Nagueh SF, Smiseth OA, Appleton CP, Byrd BF 3rd, Dokainish H, Edvardsen T, Flachskampf FA, Gillebert TC, Klein AL, Lancellotti P, Marino P, Oh JK, Alexandru Popescu B, Waggoner AD, Houston T, Oslo N, Phoenix A, Nashville T, Hamilton OC, Uppsala S, Ghent, Liege B, Cleveland O, Novara I, Rochester M, Bucharest R. St. Louis M. Recommendations for the Evaluation of Left Ventricular Diastolic Function by Echocardiography: An Update from the American Society of Echocardiography and the European Association of Cardiovascular Imaging. Eur Heart J Cardiovasc Imaging. 2016;17(12):1321-60. https://doi.org/10.1093/ehjci/jew082.

18. Nagueh SF, Smiseth OA, Appleton CP, Byrd BF 3rd, Dokainish H, Edvardsen T, Flachskampf FA, Gillebert TC, Klein AL, Lancellotti P, Marino P, Oh JK, Popescu BA, Waggoner AD. Recommendations for the Evaluation of Left Ventricular Diastolic Function by Echocardiography: An Update from the American Society of Echocardiography and the European Association of Cardiovascular Imaging. J Am Soc Echocardiogr. 2016;29(4):277-314. https://doi.org/10.1016/j.echo.2016.01.011.

19. Messina A, Pelaia C, Bruni A, Garofalo E, Bonicolini E, Longhini F, Dellara E, Saderi L, Romagnoli S, Sotgiu G, Cecconi M, Navalesi P. Fluid Challenge During Anesthesia: A Systematic Review and Metaanalysis. Anesth Analg. 2018;127(6):1353-64. https://doi.org/10.1213/ANE.0000000000003834.

20. Alvarado Sanchez JI, Amaya Zuniga WF, Monge Garcia MI. Predictors to Intravenous Fluid Responsiveness. J Intensive Care Med. 2018;33(4):227-40. DOI:10.1177/0885066617709434.

21. Burkhoff $D$. The conductance method of left ventricular volume estimation. Methodologic limitations put into perspective. Circulation. 1990;81:703-6. https://doi.org/10.1161/01.cir.81.2.703.

22. Meersch M, Schmidt C, Zarbock A. Echophysiology: the transesophageal echo probe as a noninvasive Swan-Ganz catheter. Curr Opin Anaesthesiol. 2016;29(1):36-45. https://doi.org/10.1097/AC0.0000000000000277.

23. Porter TR, Shillcutt SK, Adams MS, Desjardins G, Glas KE, Olson JJ, Troughton RW. Guidelines for the Use of Echocardiography as a Monitor for Therapeutic Intervention in Adults: A Report from the American Society of Echocardiography. J Am Soc Echocardiogr. 2015;28(1):40-56. https://doi.org/10.1016/j.echo.2014.09.009.

24. Daniel WG, Erbel R, Kasper W, Visser CA, Engberding R, Sutherland GR, Grube E, Hanrath P, Maisch B, Dennig K, et al. Safety of transesophageal echocardiography. A multicenter survey of 10,419 examinations. Circulation. 1991;83(3):817-21. https://doi.org/10.1161/01.cir.83.3.817.

\section{Figures}



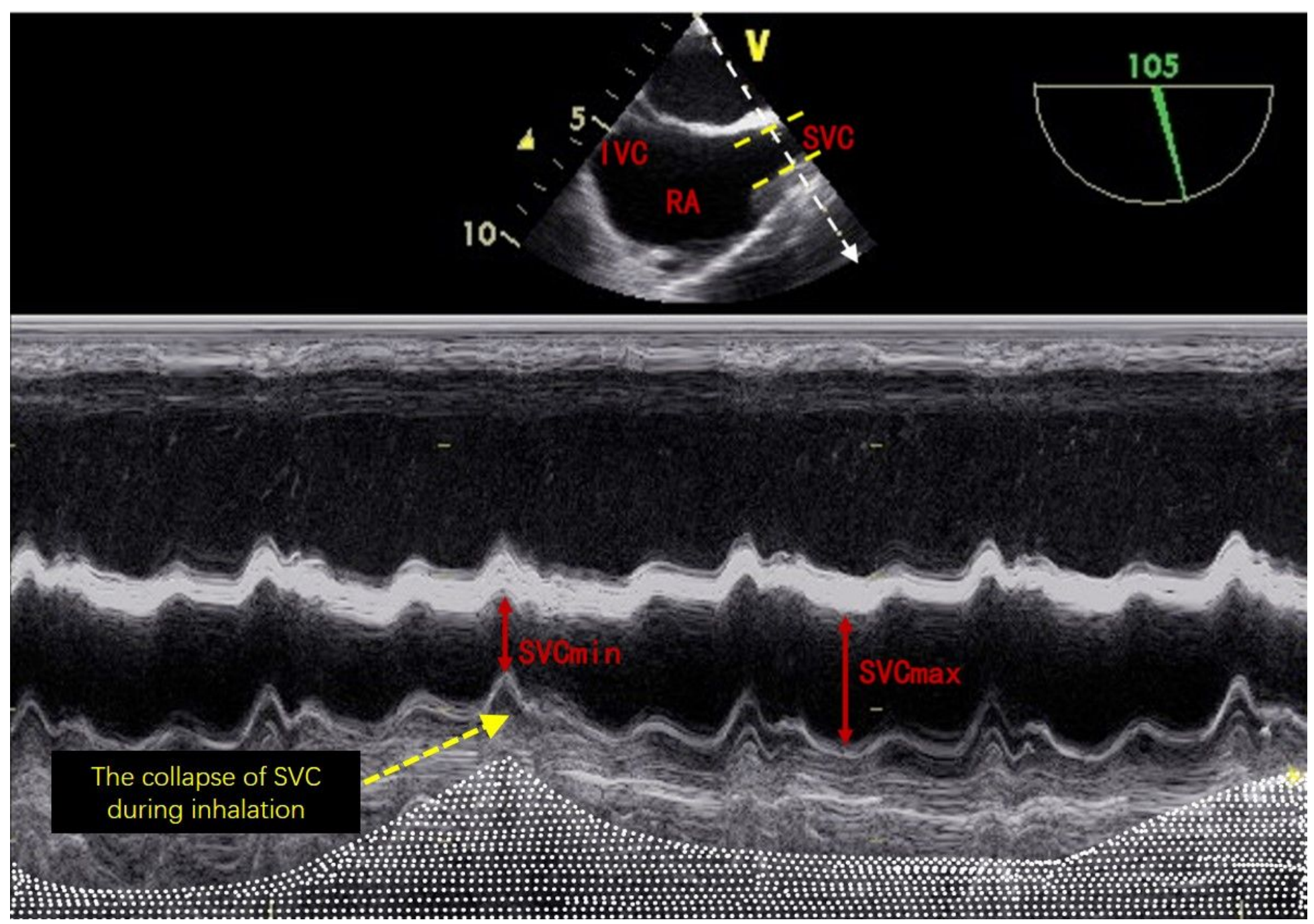

\section{Figure 1}

Measurement of the SVC diameter, take the M-mode cursor (white arrow) perpendicular to the SVC (yellow dotted line) in Mid Bicaval View. Mechanical ventilation cycle was indicated by spontaneously airway pressure wave (white dotted area). SVC superior vena cava, RA right atrial, IVC inferior vena cava, SVCmin minimum diameter of SVC, SVCmax: maximum diameter of SVC 


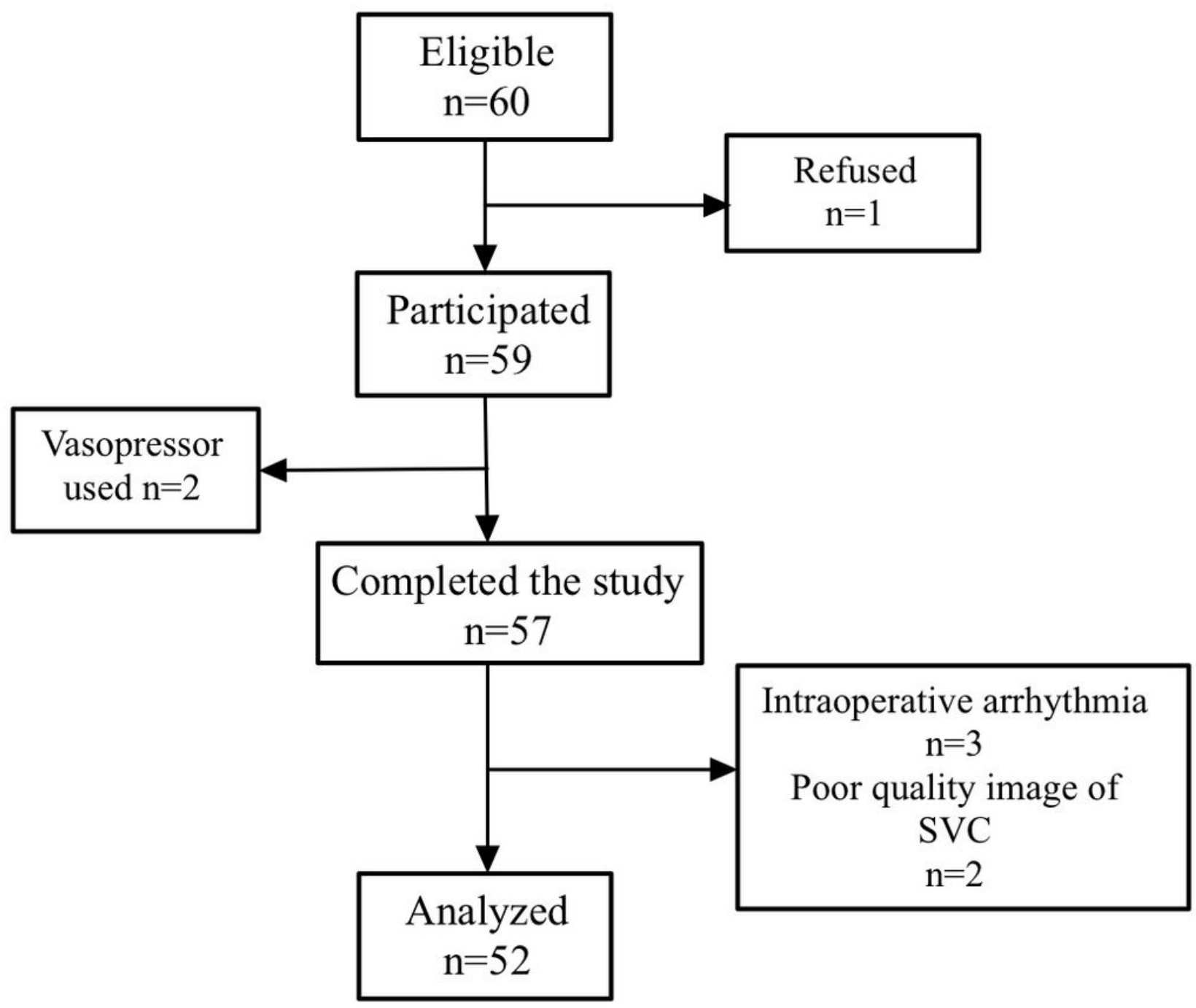

Figure 2

Flowchart of enrollment and outcomes SVC superior vena cava
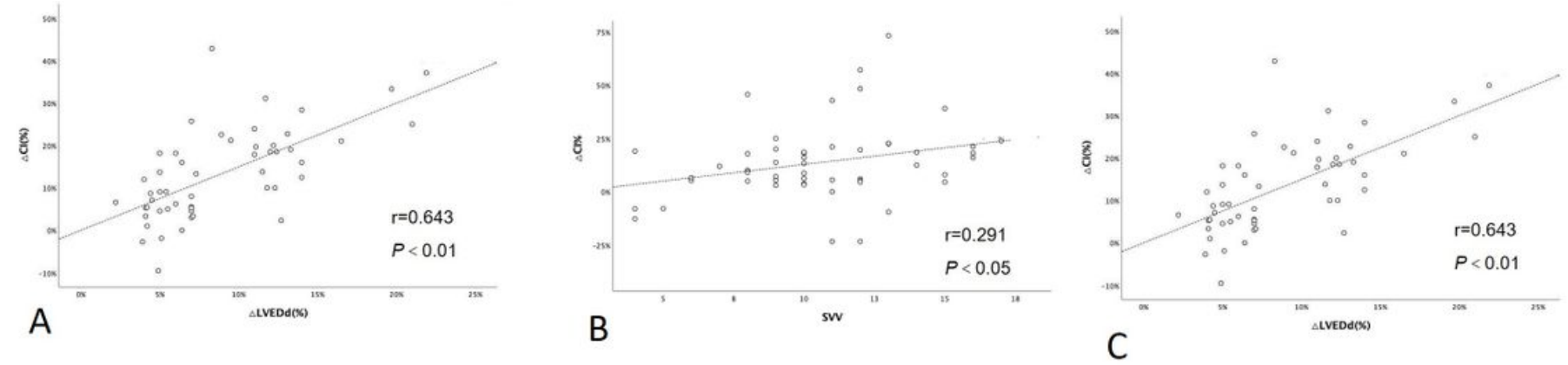

Figure 3 
A. Relationship between $\triangle \mathrm{Cl}$ and $\triangle \mathrm{LVEDd}$ in all patients $\mathrm{B}$. Relationship between $\triangle \mathrm{Cl}$ and basic SVC-Cl in all patients $\mathrm{C}$. Relationship between $\triangle \mathrm{Cl}$ and basic $S V V$ in all patients

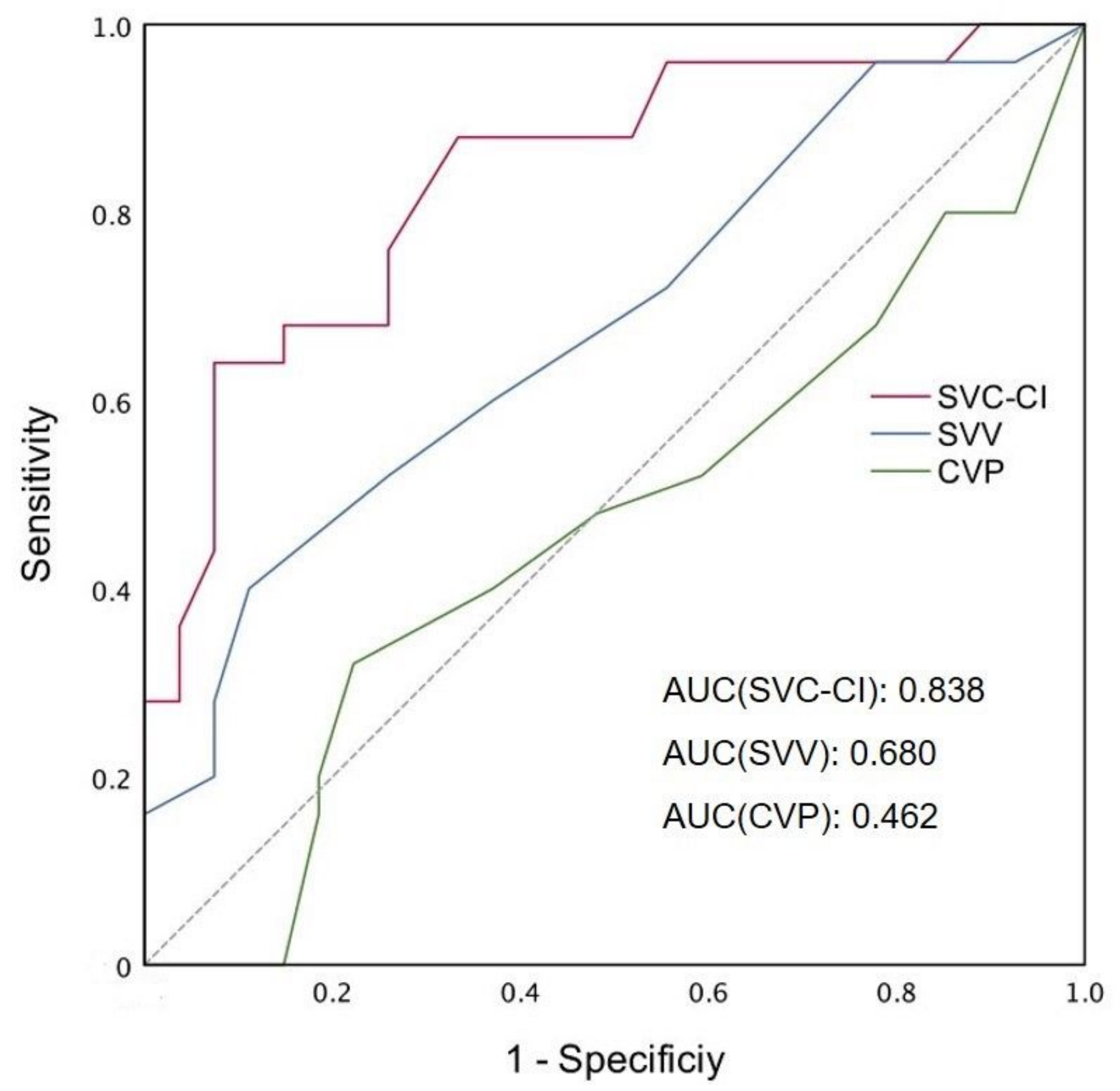

Figure 4

Area under the receiver operating characteristic (ROC) curves to predict fluid responsiveness at baseline. SVC-Cl: superior vena cava collapsibility index; SVV: stroke volume variation; CVP: central vena pressure; ROC: receiver operating characteristics 